\title{
Psychometric Properties of the Persian Montreal Cognitive Assessment in Mild Cognitive Impairment and Alzheimer Disease
}

\author{
Vahid Rashedi ${ }^{a}$ b Mahshid Foroughan ${ }^{b}$ Negin Chehrehnegar ${ }^{c}$ \\ a School of Behavioral Sciences and Mental Health (Tehran Institute of Psychiatry), Iran University of Medical \\ Sciences, Tehran, Iran; ${ }^{b}$ Iranian Research Center on Aging, The University of Social Welfare and Rehabilitation \\ Sciences, Tehran, Iran; ' Cinnaeus Centre HEAD, Swedish Institute for Disability Research, Department of Behavioural \\ Sciences and Learning, Linköping University, Linköping, Sweden
}

\section{Keywords}

Montreal cognitive assessment · Cognitive screening •

Psychometric properties · Mild cognitive impairment

\begin{abstract}
Introduction: The Montreal Cognitive Assessment (MoCA) is a cognitive screening test widely used in clinical practice and suited for the detection of Mild Cognitive Impairment (MCl). The aims were to evaluate the psychometric properties of the Persian MoCA as a screening test for mild cognitive dysfunction in Iranian older adults and to assess its accuracy as a screening test for $\mathrm{MCl}$ and mild Alzheimer disease (AD). Method: One hundred twenty elderly with a mean age of $73.52 \pm 7.46$ years participated in this study. Twenty-one subjects had mild AD (MMSE score $\leq 21), 40$ had MCl, and 59 were cognitively healthy controls. All the participants were administered the Mini-Mental State Examination (MMSE) to evaluate their general cognitive status. Also, a battery of comprehensive neuropsychological assessments was administered. Results: The mean score on the Persian version of the MoCA and the MMSE were 19.32 and 25.62 for $\mathrm{MCl}$ and 13.71 and 22.14 for AD patients, respectively. Using an optimal cutoff score of 22 the MoCA test detected $86 \%$ of $\mathrm{MCl}$ subjects, whereas the MMSE with a cutoff score of 26 detect-
\end{abstract}

ed $72 \%$ of $\mathrm{MCl}$ subjects. In AD patients with a cutoff score of 20 , the MoCA had a sensitivity of $94 \%$ whereas the MMSE detected $61 \%$. The specificity of the MoCA was $70 \%$ and $90 \%$ for $\mathrm{MCl}$ and $\mathrm{AD}$, respectively. Discussion: The results of this study show that the Persian version of the MoCA is a reliable screening tool for detection of $\mathrm{MCl}$ and early stage $\mathrm{AD}$. The MoCA is more sensitive than the MMSE in screening for cognitive impairment, proving it to be superior to MMSE in detecting $\mathrm{MCl}$ and mild $\mathrm{AD}$.

(c) 2021 The Author(s)

Published by S. Karger AG, Basel

\section{Introduction}

The ageing population has increased in the last few decades, with a corresponding rise in age-associated neurodegenerative disorders $[1,2]$. The number of people affected by dementia is increasing rapidly and it has impact on the economy, health, and community resources [3-5]. Cognitive screening measures need to be able to differentiate normal age-related cognitive changes and pathological changes in cognition [6]. Diagnosis pathological changes in cognition require tools that are easy to administer and interpret and have a high diagnostic accuracy. 
Table 1. Demographic characteristics of the study sample

\begin{tabular}{lcccc}
\hline Variable & Control $(n=59)$ & MCI $(n=40)$ & AD $(n=21)$ & $p$ value \\
\hline Age, years & $62.55(6.7)$ & $68.10(8.8)$ & $73.66(7.7)$ & $<0.001$ \\
Females, \% $(n)$ & $61(36)$ & $67.5(27)$ & $66.7(14)$ & 0.77 \\
Education, years & $11.67(5.1)$ & $7.67(5.1)$ & $8.19(6.1)$ & $<0.001$ \\
Subjective memory complains, & $67.8(40)$ & $80(32)$ & $95.2(20)$ & 0.03 \\
$\quad \%(n)$ & & & & \\
\hline
\end{tabular}

Values are presented as means (SD) unless otherwise stated. ADL, activity of daily living.

The most generally used clinical tool for cognitive assessment is the Mini-Mental State Examination (MMSE), which is not adequate for a more subtle cognitive screening, such as for mild cognitive impairment (MCI). Furthermore, the MMSE does not assess abstraction or complicated executive function abilities, which leads to its poor sensitivity in the early stages of cognitive impairment and depends on the integrity of the frontal lobe and associated structures for performing goal-directed behaviors $[7,8]$. Besides, it has been proven that executive functioning can be considered as an early subtle deficit in MCI patients before the clinical representation of the disease [9], so the executive functions are an important cognitive domain in light of an early diagnosis of dementia.

The Montreal Cognitive Assessment (MoCA) was developed by Nasreddine et al. [10] and it evaluates several cognitive domains including visuospatial ability/executive functioning, language, recall, attention, and orientation. Compared to the MMSE, the MoCA has a greater variety of subtests; thus it is probably a more sensitive screening tool to assess cognitive impairment as it takes more cognitive domains into account. Evidence shows that the MoCA distinguishes patients with MCI from the cognitively typical population and it is one of the most widely used cognitive screening tools around the world [10].

The aims of the current study were to examine the psychometric properties of MoCA and to compare its diagnostic accuracy with that of the MMSE in terms of detection of MCI in elderly in light of our current study which considers executive function as an early cognitive disturbance in MCI. We analyzed the differences between MoCA and MMSE scores with equivalent scores and cutoff values for MCI versus $\mathrm{AD}$ dementia and the contribution of domain subscores in differentiating MCI from the control group in an Iranian population.

\section{Methods}

\section{Participants}

Of these 120 participants, 21 had a clinical diagnosis of $\mathrm{AD}, 40$ were diagnosed as having $\mathrm{MCI}$, and 59 comprised the control group. The AD patients had to have an MMSE score $<22$, and the MCI subjects had to have an MMSE score $\geq 22$ and no obvious communication problems. Patients were recruited from 2 memory clinics, i.e., the Rehabilitation Hospital (Tehran, Iran) and the Brain and Cognition Clinic (Tehran, Iran). Control subjects were selected from community-dwelling older adults. The inclusion criterion for the controls was cognitive normality, as defined by a performance within 1 SD in all neuropsychological measures (Table 1). The inclusion criteria for the participants were being 60 years or older and having no salient communication problems. The Geriatric Depression Scale-15 (GDS-15) [11] with a cutoff $\leq 7$, neuropsychiatric comorbidities, cognitive therapy $<6$ months before this study, and motor, visual or auditory deficits that could influence the cognitive assessment were used as exclusion criteria. The participants with MCI were those who had memory complaints and no impairment of basic functional activities of daily living as measured by the Barthel index [12].

\section{Procedure}

All the participants underwent a battery of comprehensive neuropsychological assessments. The diagnosis of patients was based on a multidisciplinary team consensus according to the results of the comprehensive assessment and using the Petersen working group criteria for MCI [13]. The MCI group included patients classified as having amnestic MCI (single or multiple domains) with a clinical dementia rating (CDR) of 0.5 . The normal controls had a scored $\geq 24$ points on the MMSE. The AD group included only mild to moderate cases $(C D R \leq 2$ and MMSE $\geq 12)$. The control subjects had normal activities of daily living and a normal cognitive function $(\mathrm{CDR}=0$ and $\mathrm{MMSE} \geq 22)$. The $\mathrm{AD}$ participants were diagnosed according to the DSM-5 and presented significant cognitive deficits in both the MMSE and Rey Auditory Verbal Learning Test (RVALT) [14]. See Table 1 for full sample descriptive statistics.

All of the participants were recruited between January 2017 and October 2017, and the MoCA and MMSE questionnaires were administered by 2 gerontologists. The MoCA test evaluates: attention/working memory (target detection task, serial sevens, and forward and backward recall of digits) executive functions (Trails B task, phonemic fluency, and verbal abstraction), episodic memory 
Table 2. Group comparison of clinical and cognitive performance

\begin{tabular}{lcccc}
\hline Variable & Control $(n=59)$ & MCI $(n=40)$ & AD $(n=21)$ & $p$ value \\
\hline ADL & $99.57(1.68)$ & $99.12(2.47)$ & $97.61(4.06)$ & 0.055 \\
GDS & $3.93(3.31)$ & $6.20(3.91)$ & $3.00(2.30)$ & 0.13 \\
CDR & $0.09(0.10)$ & $0.23(0.19)$ & $0.70(0.67)$ & $<0.001$ \\
RVALT & $7.4(1.4)$ & $5.73(1.5)$ & $4.22(1.1)$ & $<0.001$ \\
MMSE & $28.13(1.60)$ & $25.62(3.22)$ & $22.14(3.13)$ & $<0.001$ \\
MoCA & $24.50(2.95)$ & $19.32(4.02)$ & $13.66(3.75)$ & $<0.001$ \\
\hline
\end{tabular}

Values are presented as means (SD). ADL, activities of daily living.

Table 3. Regression estimates of the relative contributions of age, education, and diagnosis to the MoCA scores

\begin{tabular}{|c|c|c|c|c|c|c|c|c|}
\hline & \multicolumn{4}{|l|}{ MCI } & \multicolumn{4}{|l|}{$\mathrm{AD}$} \\
\hline & $\begin{array}{l}\text { regression } \\
\text { coefficient }\end{array}$ & $t$ & $p$ value & $\eta^{2}$ & $\begin{array}{l}\text { regression } \\
\text { coefficient }\end{array}$ & $t$ & $p$ value & $\eta^{2}$ \\
\hline Age (years) & -0.29 & -6.36 & $<0.001$ & 0.46 & -0.18 & -2.89 & 0.006 & 0.34 \\
\hline Education & 0.81 & 3.84 & $<0.001$ & 0.28 & 0.22 & 2.10 & 0.05 & 0.25 \\
\hline
\end{tabular}

(recall task), language (naming task and sentence repetition), and visuospatial skills (clock drawing and 3-dimensional figure copy). MoCA scores range from 0 to 30 points (with the highest score indicating better function).

\section{Results}

\section{Study Participants}

The mean $( \pm \mathrm{SD})$ age of the controls, the MCI patients, and the $\mathrm{AD}$ patients was $62.55 \pm 6.78,68.10 \pm 8.81$, and $73.52 \pm 7.46$ years, respectively (Table 1 ). The clinical characteristics of the groups are summarized in Table 2. Between groups, no statistically significant difference was found in terms of gender $(p=0.77)$ or age $(p=0.06)$, though the AD group was younger. There were significant differences in educational level $(p<0.001)$ (the AD group had a lower education level)

The Cronbach $\alpha$ of the MoCA was 0.86 and that of the MMSE was 0.74. In this study, age showed a moderate negative correlation with the MoCA score $\left(r_{118}=-0.47\right.$; $p<0.001$ ), and years of education showed a moderate positive correlation with the MoCA score $\left(r_{118}=0.36\right.$; $p \leq 0.01)$. However, age and education were not significantly correlated with each other $(p=0.46)$. A regression analysis was used to identify which variables had a significant effect on the MoCA, as shown in Table 3.

Psychometric Properties of the

Persian MoCA

\section{MoCA Subscores}

A one-way ANCOVA controlling for both age and education showed a significant effect of group on MoCA performance (95\% CI 1.35-1.79; $p<0.0001$ ), and the post hoc analysis showed that performance differed significantly between all patients and healthy controls had a higher performance compared to the $\mathrm{MCI}$ and $\mathrm{AD}$ groups (95\% CI $0.7-0.81 \%$; $p<0.0001$ ). Also, a one-way ANOVA for each of the MoCA domains showed statistically significant differences in scores between all 3 groups $(p<0.001)$. The control group had a higher performance (Table 4). The 95\% CI of the MoCA subscores for each group are displayed in Figure 1. Post hoc analyses showed that the scores of the control group were significantly higher than those of the patients with MCI and mild AD $(p<0.001)$. ROC curve analysis showed that the MoCA is a more sensitive instrument than the MMSE in distinguishing individuals with MCI from normal controls (AUC $=0.85$ ) and individuals with $\mathrm{AD}$ from normal controls (AUC $=0.99$ ). According to the Youden index, the optimal cutoff for distinguishing individuals with MCI from controls for the MOCA was 22 points.

Table 5 shows AUC values for the MoCA and MMSE tests for discriminating controls from patients with MCI and mild $\mathrm{AD}$. According to the AUC, the discriminant potential of the MoCA for MCI was high, with an AUC 
Fig. 1. Point and interval estimates of the MoCA subscore. The $95 \%$ CI of the MoCA subscores in the normal controls and MCI and $\mathrm{AD}$ patients are shown.

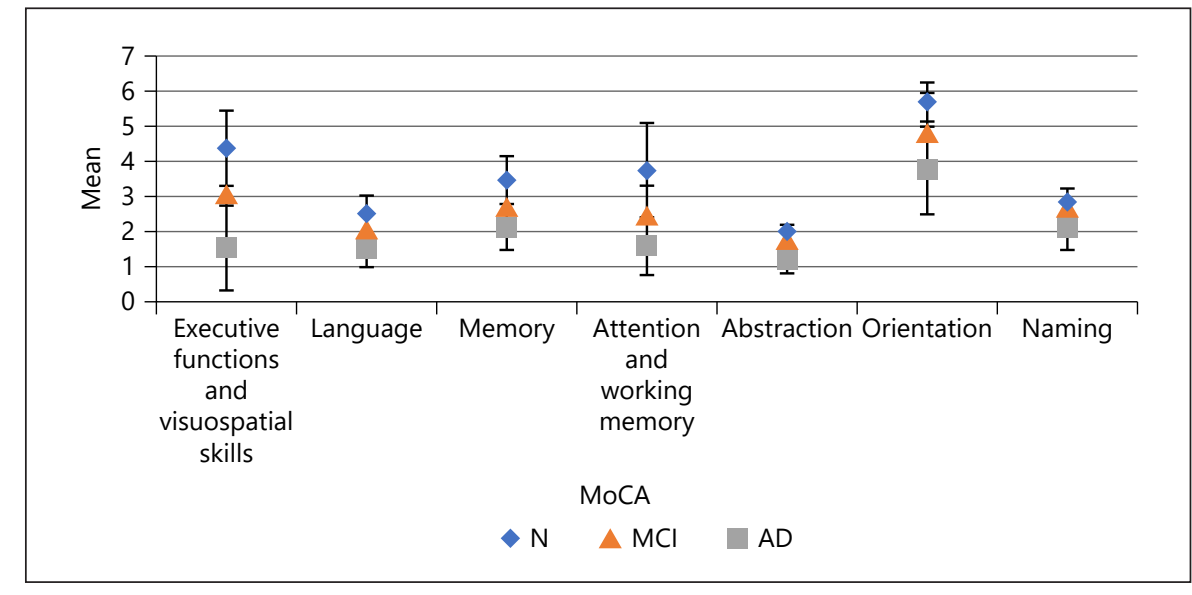

Table 4. Mean MoCA domain scores and SD and results of one-way ANCOVA controlling for age and education with 95\% CI

\begin{tabular}{|c|c|c|c|c|c|c|c|}
\hline Cognitive domains & $\begin{array}{l}\text { Normal } \\
(n=56)\end{array}$ & $\begin{array}{l}\text { MCI } \\
(n=40)\end{array}$ & $\begin{array}{l}\mathrm{AD} \\
(n=21)\end{array}$ & $p$ value $^{\mathrm{a}}$ & $\begin{array}{l}p \text { value }^{\mathrm{b}} \\
\text { (normal vs. MCI) }\end{array}$ & $\begin{array}{l}p \text { value }^{\mathrm{b}} \\
\text { (normal vs. } \mathrm{AD} \text { ) }\end{array}$ & $\begin{array}{l}p \text { value }^{\mathrm{b}} \\
\text { (MCI vs. AD) }\end{array}$ \\
\hline \multicolumn{8}{|l|}{ Executive function and } \\
\hline Language & $2.50(0.53)$ & $2.05(0.59)$ & $1.47(0.51)$ & $<0.001$ & $<0.001$ & $<0.001$ & $<0.001$ \\
\hline Memory & $3.47(0.67)$ & $2.65(0.76)$ & $2.09(0.62)$ & $<0.001$ & $<0.001$ & $<0.001$ & $<0.001$ \\
\hline Attention and working memory & $3.76(1.34)$ & $2.45(0.87)$ & $1.57(0.81)$ & $<0.001$ & $<0.001$ & $<0.001$ & $<0.001$ \\
\hline Abstraction & $1.98(0.13)$ & $1.77(0.42)$ & $1.19(0.40)$ & $<0.001$ & $<0.001$ & $<0.001$ & $<0.001$ \\
\hline Orientation & $5.71(0.55)$ & $4.82(1.15)$ & $3.76(1.26)$ & $<0.001$ & $<0.001$ & $<0.001$ & $<0.001$ \\
\hline Naming & $2.83(0.37)$ & $2.55(0.50)$ & $2.09(0.62)$ & $<0.001$ & $<0.001$ & $<0.001$ & $<0.001$ \\
\hline
\end{tabular}

${ }^{\mathrm{a}}$ ANCOVA. ${ }^{\mathrm{b}}$ Bonferroni post hoc analysis.

Table 5. Diagnostic properties of the MoCA

\begin{tabular}{lllllll}
\hline $\begin{array}{l}\text { Psychometric } \\
\text { properties }\end{array}$ & Cutoff & AUC & Sensitivity, \% & Specificity, \% & PPV, \% & NPV, \% \\
\hline $\begin{array}{l}\text { MCI } \\
\text { MoCA }\end{array}$ & $<22$ & 0.85 & 86.4 & 70.0 & 81 & 77.8 \\
MMSE & $<26$ & 0.73 & 72.9 & 67.5 & 76.8 & 62.8 \\
AD & & & & & & \\
MoCA & $<20$ & 0.99 & 94.9 & 90.5 & 96.6 & 94.9 \\
MMSE & $<22$ & 0.95 & 61.9 & 38.1 & 81.9 & 100 \\
\hline
\end{tabular}

NPV, negative predictive value; PPV, positive predictive value.

of 0.85 , and that for AD was excellent, with an AUC of 0.99 . In contrast, the corresponding values for the MMSE were 0.73 and 0.95 . Figure 2 provides a graphic visualization of the ROC curve analysis.

\section{Discussion}

The present study aimed to investigate the psychometric properties and discriminant validity of the MoCA test. This test has been widely used due to its brevity, simplicity, and reliability for diagnosing $\mathrm{MCI}$ and $\mathrm{AD}$. In this 


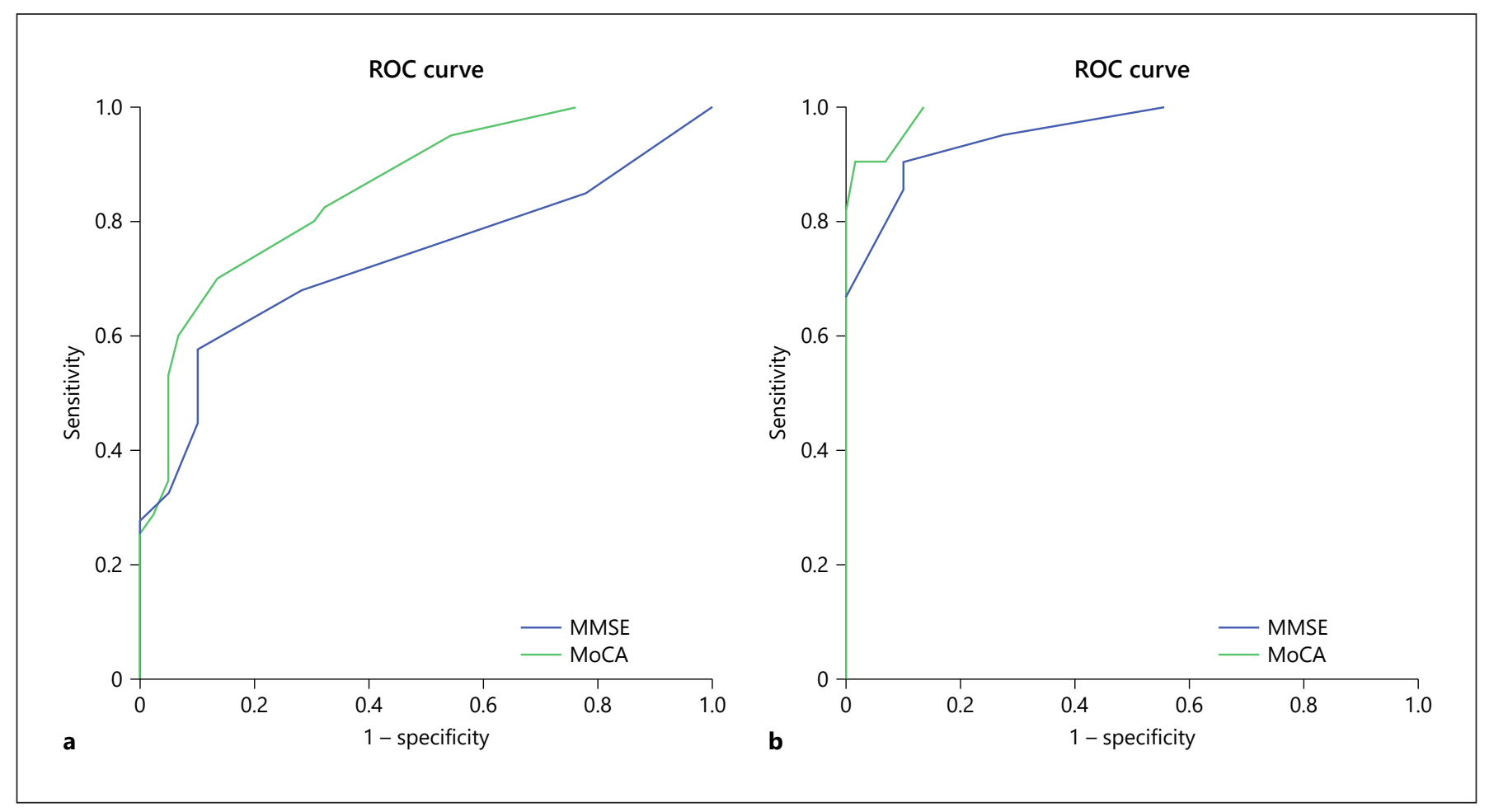

Fig. 2. ROC curves of the MoCA and the MMSE to distinguish MCI patients from normal controls (a) and AD patients from normal controls (b).

study the MoCA was evaluated in a sample of 120 individuals and compared with the MMSE. A comparison between the accuracy of the MoCA and that of the MMSE as a cognitive tracking test revealed that the MoCA has better psychometric properties. In addition to being strongly correlated with the overall MMSE score, the MoCA showed a good internal consistency, with a Cronbach $\alpha$ of 0.74 , like in previous studies [15-19]. In the current investigation, a significant statistical difference in the performance on the MoCA was found between cognitively healthy individuals, patients with MCI, and patients with mild $\mathrm{AD}$, compatible with other studies $[6,15,20$, 21].

Our results indicate, in line with previous studies [6, $22,23]$, that the Persian version of the MoCA has a high validity for detecting individuals with $\mathrm{AD}$. This result is consistent with the recognized benefits of the MoCA in detection of the early stages of cognitive impairment [1820,24 , suggesting that the MoCA is a more adequate tool for detection of MCI in the Iranian population. These findings are in consensus with studies from other countries that have revealed that, compared with the MMSE, the MoCA is more sensitive in detecting MCI in different settings and conditions [18, 22, 24]. In contrast, studies conducted in other countries have shown that the MMSE presents a low accuracy as a tracking test for $\mathrm{MCI}$ in the early stage $[25,26]$. The ROC analyses of the MoCA revealed its ability to classify patients as cognitively normal controls with acceptable sensitivity (86\%) and specificity (70\%). Compared with the MMSE, in our study the MoCA was more sensitive in detecting MCI in different settings and conditions, which was in line with previous studies $[22,24]$. These findings are in consensus with other studies from different countries that have revealed that, compared with the MMSE, the MoCA test is more sensitive in detecting MCI in different settings and conditions [18] and this justifies its clinical use. However, the cutoffs of 20 for $\mathrm{AD}$ and 22 for MCI that we found in the present study are lower than the cutoff of 26 proposed in the study of Nasreddine et al. [10] and similar studies [27-29].

The cutoff point of 26 for detecting MCI proposed in the original MoCA is highly sensitive in distinguishing MCI from normal cognitive aging, but it may increase the risk of falsely classifying cognitively normal subjects as being impaired [30]. Our attained cutoff point is in agreement with studies recommending a lower cutoff point for 
the MoCA [31, 32]. Our findings are more similar to those of Luis et al. [28], who proposed 23 as the cutoff score for the US-MoCA test, with a high sensitivity (96\%) and specificity (95\%) to detect mild cognitive deficits in the aging population. This lower cutoff point may compensate for the lower specificity with the original MoCA cutoff point [18]. Paul et al. [32] also confirmed that the original cut-off score performed normally in MRI brain scanning. Differences in the MoCA cutoff score in different subjects are probably related to age, education, and linguistic factors [33].

The MoCA consists of a few separate tasks that assess different cognitive domains [18]. It is a well-designed objective screening test that assesses different cognitive domains that are important in cognitive screening measures. [18]. More importantly, the MoCA measures an important component of dementia that's not measured by the MMSE, i.e., executive function [34]. Previously we demonstrated that executive function was one of the early domains affected in MCI and it had high sensitivity for early detection of MCI [9]. Within the subdomain evaluation, the control and MCI and $\mathrm{AD}$ groups showed statistically substantial differences in all domains. The trail-making subscale of the MoCA evaluates the integrity of a few executive functions including planning, attention, and inhibition that are related to frontal lobe impairments and the prefrontal cortex. Many studies have proven that MCI subjects are characterized by an executive disorder that presents early in the course of the disease, while the pathology does not have a clinical manifestation $[9,21]$.

Like in other studies $[35,36]$, the subjects' educational level in our study was positively correlated with the MoCA scores. The MoCA might be biased and people with a higher education would perform better. This may result from a lower cognitive reserve in less educated people and a lessened ability to recruit a neural network to compensate for age-related cognitive changes [33]. Although in some studies the level of education has been positively associated with the total MoCA score, that may be related to the cognitive reserve in more educated persons. To minimize the effect of educational levels on the MoCA score, we used education as a confounding factor in ANCOVA (Table 3). Interestingly, excluding the educational background in the ANCOVA, the mean scores on the MoCA for MCI and AD patients were still significantly different from those of the normal elderly group, which may indicate that the differences between MoCA scores in the MCI patients and controls are not a result of the educational background. Nevertheless, the educational level does not necessarily represent premorbid intellec- tual functioning, and professional status as an opportunity for many people from past generations to obtain a higher education might have been constrained by psychosocial factors such as family income [37].

Overall, this study is the first to suggest the use of the Persian MoCA for early detection of MCI. Since the MoCA assesses executive function, which is disrupted early in the development of dementia, we strongly suggest the use of the MoCA as a promising test for early detection of MCI, especially combined with other executive function tests.

\section{Acknowledgement}

The authors thank Mahdieh Esmaeili for help with data collection. We are also grateful to Dr. Mohsen Moslem, Prof. Mary Rudner, and Michaela Socher for their scientific comments and editing of this paper.

\section{Statement of Ethics}

This study was approved by the Ethics Committee of the University of Social Welfare and Rehabilitation Sciences of Tehran, Iran (ref. No. IR.USWR.REC.1395.250). Written and informed consent was provided by the participants before the examination.

\section{Disclosure Statement}

The authors declare no potential conflict of interests.

\section{Funding Sources}

No funding was received for this research.

\section{Author Contributions}

V.R.: preparation and writing of this manuscript, M.F.: project design. N.C.: writing of this paper, collection of data, and project design.

References

1 Kamalzadeh L, Moghaddamnia M, Malakouti SK, Rashedi V, Bahrampour S, Sharifi N, et al. Prevalence of Dementia Among Older Patients: A Hospital-Based Study in Iran. Am J Alzheimers Dis Other Demen. 2019;34(7-8):500-6.

2 Rashedi V, Foroughan M, Nazari H, Seeher K, Brodaty H. Validity and reliability of the Persian version of general practitioner assessment of cognition (P-GPCOG). Aging Ment Health. 2019 Aug;23(8):961-5. 
3 Zhu CW, Scarmeas N, Ornstein K, Albert M, Brandt J, Blacker D, et al. Health-care use and cost in dementia caregivers: Longitudinal results from the Predictors Caregiver Study. Alzheimers Dement. 2015;11(4):444-54.

4 Yang Z, Lin P, Levey A. Monetary costs of dementia in the United States. N Engl J Med. 2013;369(5):489-90.

5 Wimo A, Jönsson L, Bond J, Prince M, Winblad B; Alzheimer Disease International. The worldwide economic impact of dementia 2010. Alzheimers Dement. 2013 Jan;9(1):111.e3.

6 Badrkhahan SZ, Sikaroodi H, Sharifi F, Kouti L, Noroozian M. Validity and reliability of the Persian version of the Montreal Cognitive Assessment (MoCA-P) scale among subjects with Parkinson's disease. Appl Neuropsychol Adult. 2020 Sep-Oct;27(5):431-9.

7 Delgado C, Araneda A, Behrens MI. Validation of the Spanish-language version of the Montreal Cognitive Assessment test in adults older than 60 years. Neurologia. 2019 Jul Aug;34(6):376-85.

8 Brenkel M, Shulman K, Hazan E, Herrmann $\mathrm{N}$, Owen AM. Assessing Capacity in the Elderly: Comparing the MoCA with a Novel Computerized Battery of Executive Function. Dement Geriatr Cogn Disord Extra. 2017 Jul; 7(2):249-56.

9 Chehrehnegar N, Nejati V, Shati M, Esmaeili M, Rezvani Z, Haghi M, et al. Behavioral and cognitive markers of mild cognitive impairment: diagnostic value of saccadic eye movements and Simon task. Aging Clin Exp Res. 2019 Nov;31(11):1591-600.

10 Nasreddine ZS, Phillips NA, Bédirian V, Charbonneau S, Whitehead V, Collin I, et al. The Montreal Cognitive Assessment, MoCA: a brief screening tool for mild cognitive impairment. J Am Geriatr Soc. 2005 Apr;53(4): 695-9.

11 Malakouti SK, Fatollahi P, Mirabzadeh A, Salavati M, Zandi T. Reliability, validity and factor structure of the GDS-15 in Iranian elderly. Int J Geriatr Psychiatry. 2006 Jun;21(6):58893.

12 Hormozi S, Alizadeh-Khoei M, Sharifi F, Taati $\mathrm{F}$, Aminalroaya R, Fadaee $\mathrm{S}$, et al. Iranian Version of Barthel Index: Validity and Reliability in Outpatients' Elderly. Int J Prev Med. 2019;10:130.

13 Petersen RC. Mild cognitive impairment as a diagnostic entity. J Intern Med. 2004 Sep; 256(3):183-94.

14 Soltanparast S, Jafari Z, Sameni SJ, Salehi M. Psychometric properties of Persian version of the Sustained Auditory Attention Capacity Test in children with attention deficit-hyperactivity disorder. Med J Islam Repub Iran. 2014 Feb;28:14

15 Pinto TC, Machado L, Bulgacov TM, Rodrigues-Júnior AL, Costa ML, Ximenes RC, et al. Is the Montreal Cognitive Assessment (MoCA) screening superior to the Mini-Mental State Examination (MMSE) in the detection of mild cognitive impairment (MCI) and
Alzheimer's Disease (AD) in the elderly? Int Psychogeriatr. 2019 Apr;31(4):491-504.

16 Rambeau A, Beauplet B, Laviec H, Licaj I, Leconte A, Chatel C, et al. Prospective comparison of the Montreal Cognitive Assessment (MoCA) and the Mini Mental State Examination (MMSE) in geriatric oncology. J Geriatr Oncol. 2019 Mar;10(2):235-40.

17 Patnode CD, Perdue LA, Rossom RC, Rushkin MC, Redmond N, Thomas RG, et al. U.S Preventive Services Task Force Evidence Syntheses, formerly Systematic Evidence Reviews. Screening for Cognitive Impairment in Older Adults: An Evidence Update for the US Preventive Services Task Force. Rockville (MD). US: Agency for Healthcare Research and Quality; 2020.

18 Coen RF, Robertson DA, Kenny RA, KingKallimanis BL. Strengths and Limitations of the MoCA for Assessing Cognitive Functioning: Findings From a Large Representative Sample of Irish Older Adults. J Geriatr Psychiatry Neurol. 2016 Jan;29(1):18-24.

19 Chehrehnegar N, Shams F, Zarshenas S, Kazemi F. Evaluating the reliability of the Montreal Cognitive Assessment Test and its agreement with Mini Mental State Examination among healthy elderly. Eur Geriatr Med. 2013;4(4):S198

20 Chehrehnegar N, Nejati V, Shati M, Rashedi V, Lotfi M, Adelirad F, et al. Early detection of cognitive disturbances in mild cognitive impairment: a systematic review of observational studies. Psychogeriatrics. 2020 Mar;20(2): 212-28

21 Larner AJ. Screening utility of the Montreal Cognitive Assessment (MoCA): in place ofor as well as-the MMSE? Int Psychogeriatr. 2012 Mar;24(3):391-6.

22 Chehrehnegar N, Shams F, Zarshenas S, Kazemi F. Evaluating the reliability of the montreal cognitive assessment test and its agreement with mini mental state examination among healthy elderly. J Res Rehab Sci. 2011; 7(5):674-80.

23 Emsaki G, Chitsaz A, Molavi H, Asgari A. Comparing the cognitive performance in patients with Parkinson's disease and healthy people. J Res Rehabil Sci. 2012;1(1):289-97.

24 Goldstein FC, Ashley AV, Miller E, Alexeeva $\mathrm{O}$, Zanders L, King V. Validity of the montreal cognitive assessment as a screen for mild cognitive impairment and dementia in African Americans. J Geriatr Psychiatry Neurol. 2014 Sep;27(3):199-203.

25 Julayanont $\mathrm{P}$, Tangwongchai $\mathrm{S}$, Hemrungrojn S, Tunvirachaisakul C, Phanthumchinda K, Hongsawat J, et al. The montreal cognitive assessment-basic: a screening tool for mild cognitive impairment in illiterate and low-educated elderly adults. J Am Geriatr Soc. 2015 Dec;63(12):2550-4

26 Folstein MF, Folstein SE, McHugh PR. "Mini-mental state". A practical method for grading the cognitive state of patients for the clinician. J Psychiatr Res. 1975 Nov;12(3): 189-98.
27 Lee JY, Cho SJ, Na DL, Kim SK, Youn JH, Kwon M, et al.; Dong Woo Lee; Hong Jin Jeon; You Ra Lee; Maeng Je Cho. Brief screening for mild cognitive impairment in elderly outpatient clinic: validation of the Korean version of the Montreal Cognitive Assessment. J Geriatr Psychiatry Neurol. 2008 Jun; 21(2):104-10.

28 Luis CA, Keegan AP, Mullan M. Cross validation of the Montreal Cognitive Assessment in community dwelling older adults residing in the Southeastern US. Int J Geriatr Psychiatry. 2009 Feb;24(2):197-201.

29 Zhao S, Guo C, Wang M, Chen W, Wu Y, Tang W, et al. A clinical memory battery for screening for amnestic mild cognitive impairment in an elderly chinese population. J Clin Neurosci. 2011 Jun;18(6):774-9.

30 Julayanont $\mathrm{P}$, Tangwongchai $\mathrm{S}$, Hemrungrojn $\mathrm{S}$, Tunvirachaisakul C, Phanthumchinda K, Hongsawat J, et al. The Montreal Cognitive Assessment-Basic: A Screening Tool for Mild Cognitive Impairment in Illiterate and LowEducated Elderly Adults. J Am Geriatr Soc. 2015;63(12):2550-4.

31 Freitas S, Simões MR, Marôco J, Alves L, Santana I. Construct Validity of the Montreal Cognitive Assessment (MoCA). J Int Neuropsychol Soc. 2012 Mar; 18(2):242-50.

32 Paul R, Lane EM, Tate DF, Heaps J, Romo DM, Akbudak E, et al. Neuroimaging signatures and cognitive correlates of the montreal cognitive assessment screen in a nonclinical elderly sample. Arch Clin Neuropsychol. 2011 Aug;26(5):454-60.

33 Julayanont $\mathrm{P}$, Brousseau $\mathrm{M}$, Chertkow $\mathrm{H}$, Phillips N, Nasreddine ZS. Montreal Cognitive Assessment Memory Index Score (MoCA-MIS) as a predictor of conversion from mild cognitive impairment to Alzheimer's disease. J Am Geriatr Soc. 2014 Apr;62(4): 679-84.

34 Patnode CD, Perdue LA, Rossom RC, Rushkin MC, Redmond N, Thomas RG, et al. Screening for cognitive impairment in older adults: An evidence update for the US Preventive Services Task Force. Rockville: US Preventive Services Task Force Evidence Syntheses; 2020

35 Pinto TC, Machado L, Bulgacov TM, Rodrigues-Júnior AL, Costa ML, Ximenes RC, et al. Influence of Age and Education on the Performance of Elderly in the Brazilian Version of the Montreal Cognitive Assessment Battery. Dement Geriatr Cogn Disord. 2018; 45(5-6):290-9.

36 Chen KL, Xu Y, Chu AQ, Ding D, Liang XN Nasreddine ZS, et al. Validation of the Chinese Version of Montreal Cognitive Assessment Basic for Screening Mild Cognitive Impairment. J Am Geriatr Soc. 2016 Dec; 64(12):e285-90.

37 Gagnon G, Hansen KT, Woolmore-Goodwin S, Gutmanis I, Wells J, Borrie M, et al. Correcting the MoCA for education: effect on sensitivity. Can J Neurol Sci. 2013 Sep;40(5): 678-83. 